\title{
Glass transitions in bituminous binders
}

\author{
Panos Apostolidis $(\mathbb{D} \cdot$ Michael Elwardany $\cdot$ Laurent Porot $\cdot$ Stefan Vansteenkiste \\ Emmanuel Chailleux
}

Received: 19 November 2020/ Accepted: 20 May 2021 / Published online: 3 June 2021

(C) The Author(s) 2021

\begin{abstract}
The RILEM TC 272 PIM (Phase and Interphase behavior of innovative bituminous Materials)_TG1 Binder has initiated an inter-laboratory program investigating the phase and interphase behaviour of bituminous binders. Within the scope, four laboratories evaluated the low temperature properties of seven binders with differential scanning calorimetry (DSC). DSC has been accepted as a powerful tool to evaluate, among others, the glass transitions, $T_{g}$, monitoring the endothermic or
\end{abstract}

This article was prepared by a task group within RILEM TC 272-PIM: Phase and Interphase behaviour of bituminous Materials. The article has been reviewed and approved by all members of the TG.

\section{TC Membership:}

Chair: Emmanuel Chailleux (IFSTTAR),

emmanuel.chailleux@ifsttar.fr

Deputy Chair: Christiane Raab (EMPA),

christiane.raab@empa.ch.

TC Members: Adrian Andriescu (FHWA),

adrian.andriescu.ctr@dot.gov, Alexandros Margaritis

(UAntwerp), alexandros.margaritis@uantwerpen.be, Bernard

Hofko (TUWien), bernhard.hofko@tuwien.ac.at, David

Mensching (FHWA), david.mensching@dot.gov, Davide

Dalmazzo (Polito), davide.dalmazzo@polito.it, Di Wang

(TUBraunschweig), di.wang@tu-braunschweig.de, Emmanuel

Chailleux (IFSTTAR), emmanuel.chailleux@ifsttar.fr,

Hamzeh Haghshenas (Huskers),

h.haghshenas@huskers.unl.edu, Hilde Soenen (Nynas), hilde.soenen@nynas.com, Ingrid Gabrielle Do Nascimento Camargo (TUWien), ingrid.camargo@tuwien.ac.at, Jiqing Zhu (VTI), jiqing.zhu@vti.se, Kees Plug (Strukton), exothermic heat flow of a material under controlled temperature conditions. There are different ways to run the test, conventional temperature linear-DSC (TL-DSC), and temperature modulation-DSC (TMDSC). The latter has been proven as an efficient method differentiating the structural relaxation phenomena from the heat capacity. In this study, emphasis was placed on comparing the $T_{g}$ measured by TL- and TM-DSC to improve the interpretation of binder glass transitions. In this study, the scope was restrained to

kees.plug@strukton.com, Laurent Porot (Kraton), laurent.porot@kraton.com, Lucia Tsantilis (Polito), lucia.tsantilis@polito.it, Michael Elwardany (FHWA), m.elwardany.ctr@dot.gov, Michalina Makowska (Aalto), michalina.makowska@aalto.fi, Panos Apostolidis (TUDelft), p.apostolidis@tudelft.nl, Sayeda Nahar (TNO), sayeda.nahar@tno.nl, Stefan Vansteenkiste (BRRC), s.vansteenkiste@brrc.be, Virginie Mouillet (CEREMA), virginie.mouillet@cerema.fr, Xavier Carbonneau (COLAS), carbonneau@campus.colas.fr.

P. Apostolidis $(\square)$

Delft University of Technology, Delft, The Netherlands e-mail: p.apostolidis@tudelft.nl

M. Elwardany

Western Research Institute, Laramie, USA

e-mail: michael.elwardany@uwyo.edu

Present Address:

M. Elwardany

Engineering and Software Consultants, Inc. at FHWATFHRC, Washington D.C., USA 
two Polymer modified Binders (PmBs): a commercially available modified binder named PmB1 and a highly modified binder (7.5 wt \% high vinyl SBS) named PmB2, were evaluated and compared with two neat bituminous binders. Based on the thermographs of the PmB2 obtained through this inter-laboratory program, it was observed that the modification by $7.5 \%$ SBS resulted in a decrease of the $T_{g}$. This reduction of $T_{g}$ reflects the positive influence of SBS at low temperatures.

Keywords Differential scanning calorimetry · Glass transition - Bitumen - Binder - Styrene-butadienestyrene copolymer

\section{Introduction}

Differential scanning calorimetry (DSC) has been accepted as a robust device to investigate the glass transition and the amount of crystallisable and dissolvable fractions in bitumen [1-4]. Through the conventional temperature linear-DSC measurements (TL-DSC), the heat flow into and out of a sample is monitored under a controlled temperature program. Bituminous binders being complex material, the temperature modulation-DSC (TM-DSC) technique has been proven as more efficient and sensitive than the TL-DSC measurements when the structural analysis of bitumen is needed to be assessed [5]. Particularly, both the reversing and non-reversing phenomena of materials can be assessed by using the TM-DSC. A modulated temperature signal superimposed on a linear heating or cooling program can be applied to elucidate the thermo-kinetic phenomena occurring through thermal scanning [6-9]. The total

\section{Porot}

Kraton Polymers B.V., Almere, The Netherlands e-mail: laurent.porot@kraton.com

S. Vansteenkiste

Belgian Road Research Centre, Brussels, Belgium

e-mail: s.vansteenkiste@brrc.be

E. Chailleux

Université Gustave Eiffel, Bougenais, France

e-mail: emmanuel.chailleux@univ-eiffel.fr
MT-DSC signals can be split into reversible and nonreversible signals. The latter two can be used to decompose the time-dependent thermal non-equilibrium transitions from the equilibrium-in-nature transformations [10, 11]. Thus, high sensitivity and resolution analyses can be performed using a modulated signal in TM-DSC, and the heat capacity $\left(C_{p}\right)$ can be measured, providing accurate glass transition analyses. As it is less uncertain and ambiguous the definition and selection of baselines and endpoint in TM-DSC than in TL-DSC, the detection of different thermal events and enthalpy changes is more precise $[5,12-14]$.

Over the years, various theories have been introduced to explain the nature of bitumen, from being a natural polymer consisted of repeating units [15], to a binder of oligomeric structure [16], and a colloidal biphasic system of asphaltenes and maltenes [17]. However, according to [18], no hypothetical microstructure of bitumen exists. The physical and mechanical properties of bitumen originate from the intermolecular interactions among its constituents that are difficult to be identified due to their high complexity. Indeed, predictions of the thermal behavior of bitumen are not straightforward, unlike other systems. The calorimetric behavior of bitumen resembles that of other hydrocarbons [19, 20]. Hence, special attention should be paid to interpreting the nature of bitumen by the intermolecular interactions between maltenes and asphaltenes [21].

Recent studies on the thermal behavior of bitumen via TM-DSC have shown that the polymer-like rheology of bitumen is due to the gel-like microstructures formed by waxes without repeated groups in bitumen [10]. The presence of waxes in bitumen attributes the melting peaks [22] and the crystallisation upon heating due to limited mobility, associated with non-crystallisable compounds during cooling [20]. In addition to enthalpic events, the glass transition region of bituminous binders extends over a wide temperature range, similarly to polymer-like materials [23], ranging between -60 and $0{ }^{\circ} \mathrm{C}$ [2], or between -50 and $-10{ }^{\circ} \mathrm{C}$ [24]. The $T_{g}$ is related to the overall chemical composition of bitumen and has been considered to determine the source, the process, and aging level of bitumen [1-4]. The width of the glass transition region increases with higher asphaltenes content and higher oxidation levels [3, 4]. 
In general, polymers behave as amorphous materials at temperatures above the $T_{\mathrm{g}}$, while below the $T_{\mathrm{g}}$, they behave like glass. In the transition region from an amorphous to a glassy state, a stepwise increase of modulus, specific volume, and thermal expansion coefficient happen. The glass transition region is the range of temperatures that correspond to this transition. Binder rheological behavior is defined by three regions: the near glass region below the $T_{\mathrm{g}}$, terminal region above the crossover temperature, and an intermediate "transition" region between them [25]. Rheological measurements can be used to determine the $T_{g}$ of binder, which is defined as the temperature that corresponds to the maximum loss moduli at a given reference frequency $[17,25]$. From the mechanics point of view, the $T_{g}$ can help understand the thermal cracking and the performance of various binders at low temperatures [26, 27]. Recent studies based on asphalt bending beam testing, acoustic emissions, and finite element analysis provide evidence that asphalt can be damaged on cooling even when no external restraint is imposed on the specimens. It has been theorised that the differential contraction between mastics and the surrounding aggregate develops localised tensile stresses that are defined in what is called the Internal Restraint Damage Mechanism [28]. This internal restraint damage mechanism is dependent on the binder coefficient of thermal contraction (CTC), binder rheological properties and ability to relax stresses, and binder strength [29]. All the aforementioned three key properties are highly dependent on the binder glass transition. For instance, the $T_{g}$ affects the accumulation of thermal stresses build-up in the pavement over a certain thermal history. Below the $T_{g}$, asphalt binders exhibit brittle behavior while they are ductile at temperatures above $T_{g}$.

For bituminous binders with various inclusions, such as polymers or liquid additives, the use of DSC has been proved as a robust characterisation tool to evaluate the compatibility of individual components of produced blends. The compatibility, or lack of it, determines the durability and the performance properties of bituminous blends. In polymer modifications, a single $T_{\mathrm{g}}$, otherwise a single blend-average $T_{\mathrm{g}}$, indicates a single-phase system. On the other hand, blends of two $T_{\mathrm{g}}$ values manifest a system of two phases. In bituminous binders, alterations of glass transitions in blends manifest to changes of an always single $T_{g}$. Many studies have been performed to evaluate the miscibility of various polymers in binders with the ultimate goal to link with other performance properties [12, 30-35].

An inter-laboratory experimental program was initiated within the RILEM TC 272 PIM (Phase and Interphase behaviour of innovative bituminous Materials)_-TG1 Binder. It had a broad experimental plan evaluating seven binders in two groups, one for polymer modification and one for liquid additives [36]. Over the 17 participating laboratories, four of them performed thermal analyses on bituminous binders in DSC. Special emphasis was given on comparing the values of $T_{g}$ measured by TL- and TMDSC techniques implementing different protocols to highlight the numerous different output signals to improve the interpretation of glass transitions in bituminous binders. The advantages of various calorimetric methodologies are not universally acknowledged, with slightly different temperature profiles generated by different scanning protocols lacking comprehensive understanding. The four laboratories, having performed DSC, were the Belgian Road Research Centre (Belgium), Delft University of Technology (The Netherlands), Kraton Polymers (The Netherlands), and Western Research Institute (USA). Two of them performed temperature linear DSC, and the two others temperature modulated DSC (Table 1). The name of each lab was according to the labelling of whole TG1 experimental program for which only Lab1, Lab2, Lab5 and Lab12 performed DSC.

\section{Materials}

The scope of the study was restrained to two polymer modified binders and compared with two neat bituminous binders. Two paving grade bitumen of classes

Table 1 List of participating laboratories

\begin{tabular}{ll}
\hline Laboratory & Equipment \\
\hline Lab1 & Q2000, TA \\
Lab2 & Q2000, TA \\
Lab5 & DSC, PerkinElmer \\
Lab12 & DSC2 STARe with TOPEM, Mettler Toledo \\
\hline
\end{tabular}


$35 / 50$ and 70/100 according to EN 12,591, named as Bit1 and Bit 2 respectively, were used in this study as control binders. A commercial standard polymer modified bitumen, PmB1, and a highly polymer modified bitumen, PmB2, were included. The PmB1 was plant produced, and the PmB2 was lab-produced using the Bit 2 and $7.5 \%$ of a high vinyl linear block styrene-butadiene-styrene (SBS) copolymer. Table 2 provides the basic properties of the four binders.

\subsection{Calorimetric methods}

As mentioned earlier, the heat capacity at the glass transition changes significantly, reflecting a material transition from a glassy to an amorphous viscoelastic state. Thus, the glass transition in bituminous binders is crucial when materials are designed and developed to withstand thermal stresses and subsequently mitigate cracking susceptibility at low temperatures.

Through the calorimetric measurements with DSC, the heat flow signal $[\mathrm{mW}$ or $\mathrm{mJ} / \mathrm{s}]$ typically plotted versus temperature or time, consists of two parts: (i) the heat flow needed to raise the sample temperature at the programmed rate, which is directly linked to the intrinsic heat capacity of the sample, and (ii) the heat flow arising from kinetic processes that may occur. Phase change transitions, such as melting and crystallisation, and second-order transitions, such as glass transition and its associated enthalpy relaxation, are exemplary kinetic phenomena. Therefore, the heat flow is often expressed as

$\frac{d Q}{d t}=m C_{P} \beta+f(t, T)$

where $m$ is the mass of sample, $C_{p}$ is the (temperaturedependent) specific heat capacity of sample, $\beta$ is the heating rate, and $f(T, t)$ is the heat flow due to the kinetic phenomena. The $C_{p}$ represents the heat capacity of sample of constant microstructure (equilibrium).

In this study, the heat flow curves of bituminous binders have been obtained via performing TL-DSC and TM-DSC scans. The measured heat flow is a function of the heating or cooling rates, sample heat capacity, and endothermic or exothermic events occurring in the sample. Attention was paid on the sample preparation by extracting a representative amount from the bulk material to avoid the undesired hardening due to oxidation.

A schematic representation of $C_{p}$ curves around glass transition is illustrated in Fig. 2. The increase of $C_{p}$ takes place during the transition from that of glass to an amorphous state.

Furthermore, heat transfer phenomena play a crucial role in having accurate calorimetric measurements. The larger the mass of the sample, the greater the heat flow signal under a certain heating or cooling rate. If the mass of the sample is large, then nonuniform heat distribution is obtained, resulting in a significant thermal lag between the sample and programmed temperature.

In this RILEM initiative, all the four participating laboratories used samples of weight ranging from 5 to $15 \mathrm{mg}$ to compromise between maximising the heat flow signal and minimising the thermal lag. After sealing the samples in aluminum pans, annealing was conducted before any measurement to remove any polar association existed in binders due to their thermal history. All labs calibrated the temperature scale using a pure calibrant (i.e., indium) with a wellknown $T_{g}\left(156.6^{\circ} \mathrm{C}\right)$ under a certain heating rate. All measures of heat flow were performed comparing with an empty reference cell.

For each scan, the derivative of heat flow was determined for further analysis. While the heat flow increases constantly, the derivative is null. With glass transition, the heat flow rate will pass by the inflection point, where the derivative will reach a maximal absolute value. Table 3 provides the $T_{g}$ values of samples as determined for each lab. The temperature corresponds to the maximum of the derivative signal (i.e., TL-DSC: heat flow signal, TM-DSC: heat capacity signal).
Table 2 Properties of binders

Average value from $* 6$ to 8 labs, ** 2 to 3 labs

\begin{tabular}{lllr}
\hline Sample & Penetration value* at $25^{\circ} \mathrm{C}[0.1-\mathrm{mm}]$ & Softening point* temperature $\left({ }^{\circ} \mathrm{C}\right)$ & $\mathrm{PG}^{* *}$ \\
\hline Bit1 & 40 & 54.0 & $70-22$ \\
Bit2 & 79 & 46.2 & $64-22$ \\
PmB1 & 40 & 73.8 & $76-16$ \\
PmB2 & 34 & 90.6 & $94-28$ \\
\hline
\end{tabular}


Table 3 Interlaboratory results on studied binders through heating calorimetric scans

\begin{tabular}{lllll}
\hline Sample & \multicolumn{4}{l}{ Glass transition temperature, $T_{g}\left({ }^{\circ} \mathrm{C}\right)$} \\
\cline { 2 - 5 } & Lab1 & Lab2 & Lab5 & Lab12 \\
\hline Bit1 & -13.3 & -10.5 & -16.6 & -14.5 \\
Bit2 & -22.5 & -14.4 & -23.3 & -18.3 \\
PmB1 & -23.2 & -16.9 & -25.1 & -16.8 \\
PmB2 & -25.7 & -19.2 & -25.0 & -22.0 \\
\hline
\end{tabular}

\subsection{Linear temperature protocols}

For the standard linear calorimetric scans (TL-DSC), two laboratories provided results of binder samples. Based on the calorimetric measurements of these two laboratories, i.e., Lab1 and Lab4, the $T_{g}$ values of samples were determined by calculating the maximum of the 1st derivative of the heating heat flow curve. Nevertheless, different DSC tools were used, and different scanning protocols were implemented below.

In TL-DSC applied by Lab1 (DSC Q2000, TA), the samples were annealed in DSC by preheating to $165{ }^{\circ} \mathrm{C}$ for $5 \mathrm{~min}$. The samples were immediately subjected to controlled cooling to $-60{ }^{\circ} \mathrm{C}$ with a rate of $2{ }^{\circ} \mathrm{C} / \mathrm{min}$, without modulation. They kept for $5 \mathrm{~min}$ at $-60{ }^{\circ} \mathrm{C}$ and then heated to $165{ }^{\circ} \mathrm{C}$ with the same rate of $2{ }^{\circ} \mathrm{C} / \mathrm{min}$ (average measuring rate: 1 data point per $0.7 \mathrm{~s}, \&$ plotting rate: 1 data point per $14.8 \mathrm{~s}$ ).

In TL-DSC applied by Lab12 (DSC2 STARe system equipped with M-DSC TOPEM, Mettler Toledo), the samples were heated from 25 to $130{ }^{\circ} \mathrm{C}$ with $20{ }^{\circ} \mathrm{C} / \mathrm{min}$ rate to establish sufficient contact with the bottom of the pan. Samples were kept at $130{ }^{\circ} \mathrm{C}$ for $1 \mathrm{~min}$ and then cooled down to $-80{ }^{\circ} \mathrm{C}$ with a rate of $10{ }^{\circ} \mathrm{C} / \mathrm{min}$. After $1 \mathrm{~min}$ at $-80{ }^{\circ} \mathrm{C}$, samples were heated to $160{ }^{\circ} \mathrm{C}$ with $10{ }^{\circ} \mathrm{C} / \mathrm{min}$ rate and kept at this temperature for $1 \mathrm{~min}$ to erase their thermal history. Afterward, a cooling rate of $10{ }^{\circ} \mathrm{C} / \mathrm{min}$ was applied to samples to reach $-80{ }^{\circ} \mathrm{C}$, and the sample was kept at this temperature for $1 \mathrm{~min}$. Then samples were heated to a range where cold crystallisation occurred, particularly to $0{ }^{\circ} \mathrm{C}$ with $10{ }^{\circ} \mathrm{C} / \mathrm{min}$ rate, and kept there for 5 min to facilitate the relaxation of possible occurring stresses. Samples were cooled to $-80{ }^{\circ} \mathrm{C}$ with a rate of $10{ }^{\circ} \mathrm{C} / \mathrm{min}$ and, after $1 \mathrm{~min}$ at this temperature. In the last step, samples were heated up to $160{ }^{\circ} \mathrm{C}$ with
$20{ }^{\circ} \mathrm{C} / \mathrm{min}$. The $T_{g} s$ of samples were determined while using the heating curve in this last step (average measuring rate: 1 data point per $1 \mathrm{~s}$ ).

Comparing the protocols implemented by Lab1 and Lab12, the most significant difference was only the number of steps before applying the $T_{g}$ detection measurement. Lab12 selected a faster heating rate $\left(20^{\circ} \mathrm{C} / \mathrm{min}\right)$ in the last step than Lab1 to improve the sensitivity of calorimetric measurements, minimising the appearance of thermodynamically metastable phases as cold crystallisation. The glass transition is highly dependent on the heating rate, as the frequency dependency of rheological properties of binders. A lower heating rate as implemented by Lab1 $\left(2{ }^{\circ} \mathrm{C} / \mathrm{min}\right)$ could reduce thermal lag and non-uniform temperature distribution, offering a set of data that can be compared with TM-DSC protocols of the same heating rate.

\subsection{Modulated temperature protocol}

The glass transition in studied binders was evaluated using the TM-DSC techniques as well. Temperature modulation was only necessary when analysing binders to provide high accuracy in detecting the glass transition region. In TM-DSC scans, the most commonly implemented program is the sinusoidal temperature modulation, with specified frequency and amplitude, along with a certain heating and/or cooling rate. To be more specific, a periodic temperature change is superimposed on the linear temperature ramp yielding a heating (or cooling) profile with continuously non-linearly alterations of the average temperature of a sample with time. In Fig. 1, the blue and red lines demonstrate the underlying temperature and heat flow, respectively. This method permits the simultaneous calculation of the heat capacity of the sample. Based on a Discrete Fourier Transformation, the measured amplitudes of modulation of temperature and heat flow are compared to a reference signal of the same frequency, calculating the heat capacity. A schematic representation of $C_{p}$ curves around glass transition is illustrated in Fig. 2. The increase of $C_{p}$ occurs during the transition from that of glass to amorphous in a sigmoidal way. Hence, an equivalent endothermic heat flow increase corresponds to the first term of Eq. 1.

TL-DSC scans annealing first at $165^{\circ} \mathrm{C}$ for $5 \mathrm{~min}$ to remove the thermal history. Modulated cooling and 

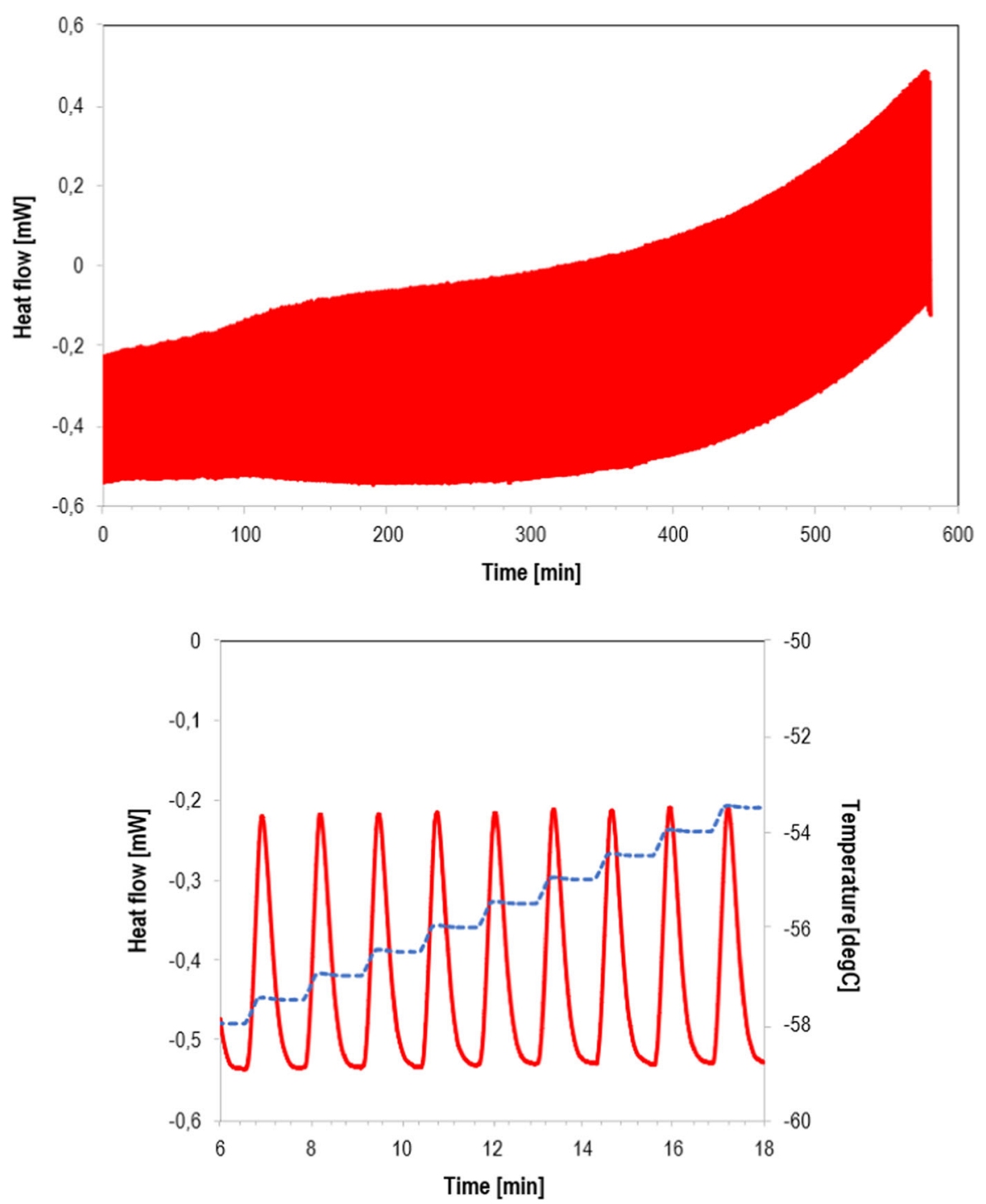

Fig. 1 Typical TM-DSC profile and heat flow generated versus time in calorimetric scans (top) and a detailed graph of TM-DSC profile with the blue dotted line and orange line to demonstrate the underlying temperature and heat flow respectively (bottom)

heating rates were then imposed on the samples using a superimposed periodic sine wave temperature perturbation. Lab2 and Lab5 applied two modulation methodologies.

In TM-DSC applied by Lab2 (DSC Q2000, TA), samples were equilibrated at $165{ }^{\circ} \mathrm{C}$ for $5 \mathrm{~min}$ and then cooled to $-90{ }^{\circ} \mathrm{C}$ at $5{ }^{\circ} \mathrm{C} / \mathrm{min}$ with $\pm 0.5^{\circ} \mathrm{C}$ modulation every $80 \mathrm{~s}$. The samples were then equilibrated for $5 \mathrm{~min}$ and then heated to $165^{\circ} \mathrm{C}$ at $2{ }^{\circ} \mathrm{C} / \mathrm{min}$ with the same modulation (average measuring rate: 1 data point per $1.2 \mathrm{~s}$ ). The DSC tool used by Lab2 provided the total and the two individual heat flow components of scanned samples. Moreover, in TM-DSC applied by Lab5 (DSC, PerkinElmer), samples cooled rate of $2{ }^{\circ} \mathrm{C} / \mathrm{min}$ to $-60{ }^{\circ} \mathrm{C}$, equilibrated at $-60{ }^{\circ} \mathrm{C}$ for $5 \mathrm{~min}$ and then heated to $165^{\circ} \mathrm{C}$ at $2{ }^{\circ} \mathrm{C} / \mathrm{min}$, with $0.5{ }^{\circ} \mathrm{C}$ modulation every $60 \mathrm{~s}$ (average measuring rate: 1 data point per $1 \mathrm{~s}$ ). All the provided $T_{g}$ values from both TM-DSC protocols were determined as the temperature at the half-height between the heat capacity of onset and endpoint of the glass transition region, as shown in Fig. 2. 


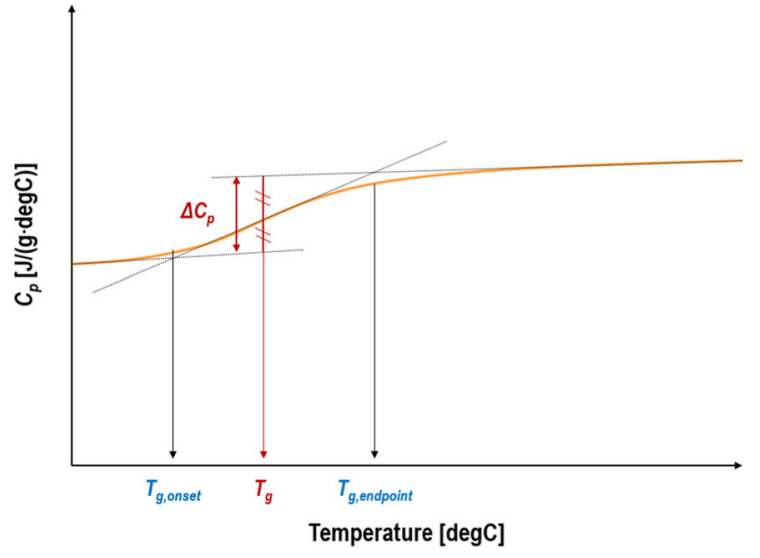

Fig. 2 Schematic of specific heat capacity change during cooling at glass transition indicating the glass transition behavior of a bituminous binder

\section{Results and discussion}

\subsection{TL-DSC}

In Fig. 3, the TL-DSC thermographs, from Lab1, of heat flow and its 1 st derivative of Bit 1 and Bit 2 binders are shown during heating (left) and cooling (right) scans, with their $T_{g}$ values to be almost the same between the labs. Similarly, the thermographs of PmB1 and PmB2 binders generated by Lab1, with the same protocol, are shown in Fig. 4. There is a slight reduction in the baseline of heat flow after the glass transition, or the appearance of a second peak of the 1st derivative of heat flow in PmB1, resulting from the cold crystallisation. In terms of physics, this second reduction accounts for the loss of molecular mobility as the microstructure of PmB1 is ordered from a glassy to a viscoelastic binder. Despite the cold crystallisation after the glass transition of PmB1, the PmB2 binder does not demonstrate a second peak of the 1st derivative of heat flow or any deviation on the baseline of heat flow signals after the glass transition. With the TL-DSC from Lab1, the $T_{g}$ values of PmB1 and PmB2 were -23.2 and $-25.7^{\circ} \mathrm{C}$, respectively. On the other hand, the TL-DSC thermographs from Lab12 have shown different $T_{g}$ values of PmB1 $\left(-16.8{ }^{\circ} \mathrm{C}\right)$ and PmB2 $\left(-22.0^{\circ} \mathrm{C}\right)$, mainly due to the difference in applied heating rate (see Fig. 5). Even though the Lab12 TL-DSC protocol has been optimized to avoid such thermodynamic metastable processes during scanning, cold crystallisation still occurred.
All the discussed $T_{g}$ values of binders determined at the peak of the 1st derivative of thermographic signals during heating are recorded in Table 3. The TM-DSC $T_{g}$ values of binders are provided in the same table and discussed in the following sub-section.

\section{TM-DSC}

A typical TM-DSC thermograph of studied binders is illustrated in Fig. 6. The glass transition region is quite apparent in the total heat flow signals, heat capacity, and reversing heat capacity versus temperature. In TM-DSC from Lab2, the total heat flow, as indicated in Fig. 6, was calculated as the moving average of the raw modulated heat flow signal. The reversing heat capacity of the total heat flow was calculated by converting the measured heat capacity into the heat flow of Eq. 1 ( $\beta$ is the average heating rate of calorimetric measurement). The non-reversing component of heat flow is the arithmetic difference between the total heat flow and heat capacity. In other words, the reversing and non-reversing heat flow were deconvoluted from the modulated heat flow through heating along with the total heat flow of TM-DSC and TL-DSC scans.

The $T_{g}$ values of samples provided by TM-DSC (Table 3) were determined based on the sigmoidal change of specific (mass normalized) heat capacity from reversing component as a step in the glass transition region. For illustration purposes, Figs. 7 and 8 show the total heat flow, heat capacity, and reversing heat capacity signals of neat and modified binders, respectively, from Lab2. The 1st derivative of those curves was plotted in the same figures to detect the glass transition. It should be noticed that the reversing heat capacity signals of PmB1 had no two peaks of its 1 st derivative as observed in TL-DSC heating scans. The effect of cold crystallisation was minimised by the TM-DSC technique in both protocols allowing the separation of structural relaxation phenomena from the total heat capacity measurements. The $T_{g}$ values recorded using the TM-DSC from Lab2 were slightly higher than of TM-DSC from Lab5. Overall, the glass transition detection based on the reversing component of heat capacity from the TM-DSC heating scans was not significantly influenced by the structural relaxation due to the underlying heating. 

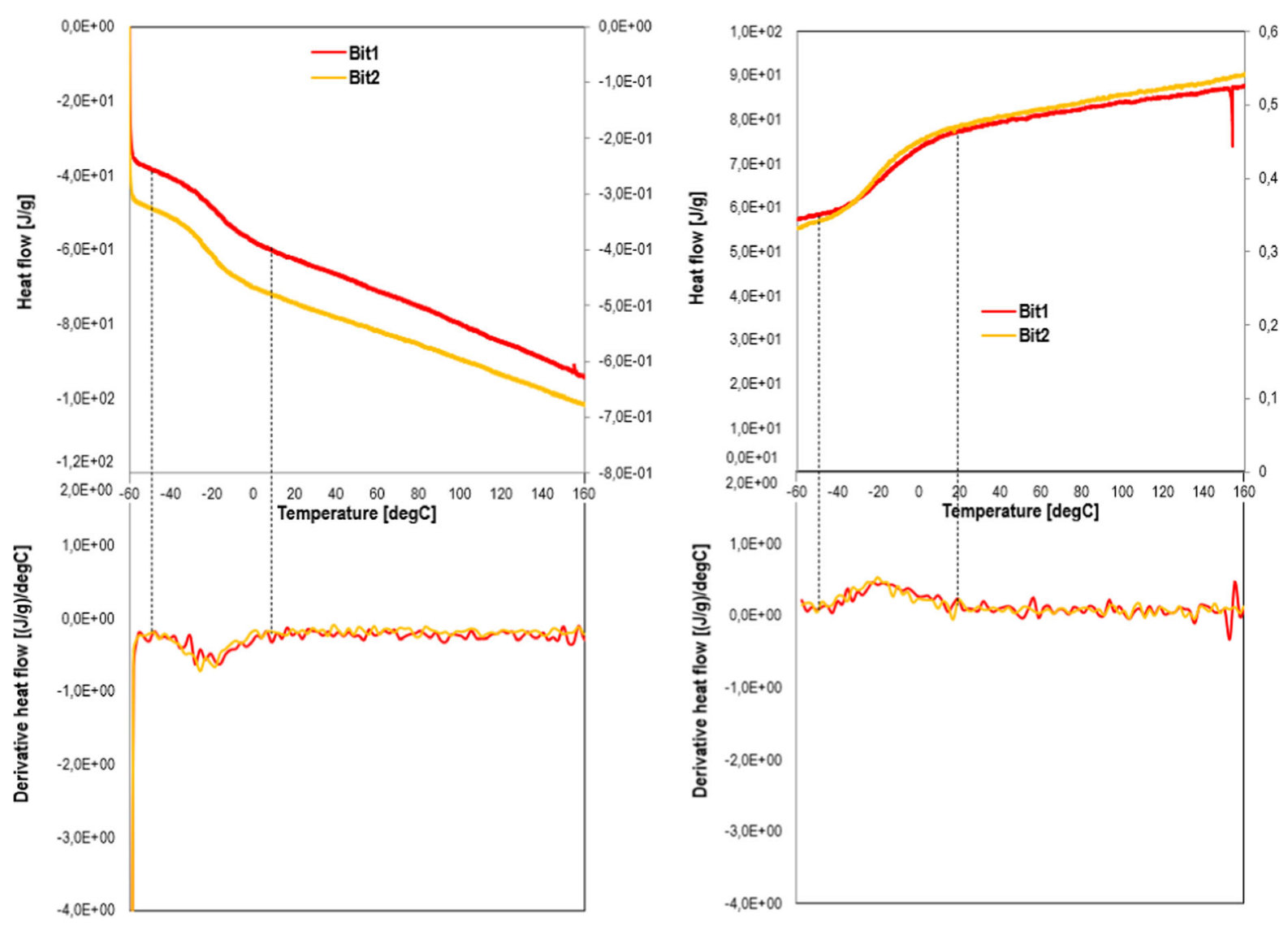

Fig. 3 Graphs of heat flow together with their derivative curves of Bit1 and Bit2 during linearly heating (left) and cooling (right) calorimetric scans (TL-DSC, Lab1)

\subsection{Effect of SBS polymer on glass transition of binders}

The high vinyl SBS copolymer, consisting of polybutadiene and polystyrene blocks, was blended with Bit2 to produce PmB2. The $T_{g}$ values of Bit2 and PmB2 generated by the different protocols were discussed above. The inclusion of $7.5 \%$ of the vinyl SBS copolymer led to the reduction of $T_{g}$. As mentioned, the $T_{g}$ change can be used as an indicator of compatibility among the components of a system. Hence, DSC measurements were performed on the pure polymer, and its glass transition was compared with Bit2 and PmB2.

Figure 9 shows the thermographs of SBS polymer, Bit2, and PmB2 generated by applying the TL-DSC: (Lab1) through $2{ }^{\circ} \mathrm{C} / \mathrm{min}$ of cooling and heating rate. The polybutadiene part of the SBS copolymer exhibited a sharp glass transition at $-47{ }^{\circ} \mathrm{C}$. A glass transition near $85^{\circ} \mathrm{C}$ can be seen in the orange curves of Fig. 9, which may be attributed to the polystyrene part of the copolymer. With $7.5 \%$ of SBS polymer in Bit2, the $T_{g}$ values were shifted from -22.5 to $25.7{ }^{\circ} \mathrm{C}$. The $T_{g}$ reduction directly reflects the actual influence of polybutadiene of SBS on bitumen, manifesting the generation of a more resistant binder against low temperature fracture.

Considering the fact that the PmB2 binder consists $7.5 \%$ of linear high vinyl SBS copolymer $\left(T_{g, S B S}:-\right.$ $\left.47{ }^{\circ} \mathrm{C}\right)$ and $92.5 \%$ of Bit $2\left(T_{g, \text { Bit } 2}:-22.5^{\circ} \mathrm{C}\right)$, then according to Fox equation [37] expressed as

$\frac{1}{\mathrm{~T}_{g, P m B}}=\frac{w_{S B S}}{\mathrm{~T}_{g, S B S}}+\frac{w_{B i t}}{\mathrm{~T}_{g B i t}}$

üthe predicted $T_{g}$ value of $\mathrm{PmB} 2\left(T_{g, P m B 2}\right)$ is $23.4{ }^{\circ} \mathrm{C}$, which is slightly higher than the actual $T_{g}$ value $\left(25.7^{\circ} \mathrm{C}\right)$ determined based on the TL-DSC (Lab1). Thus, a slight positive deviation between the 

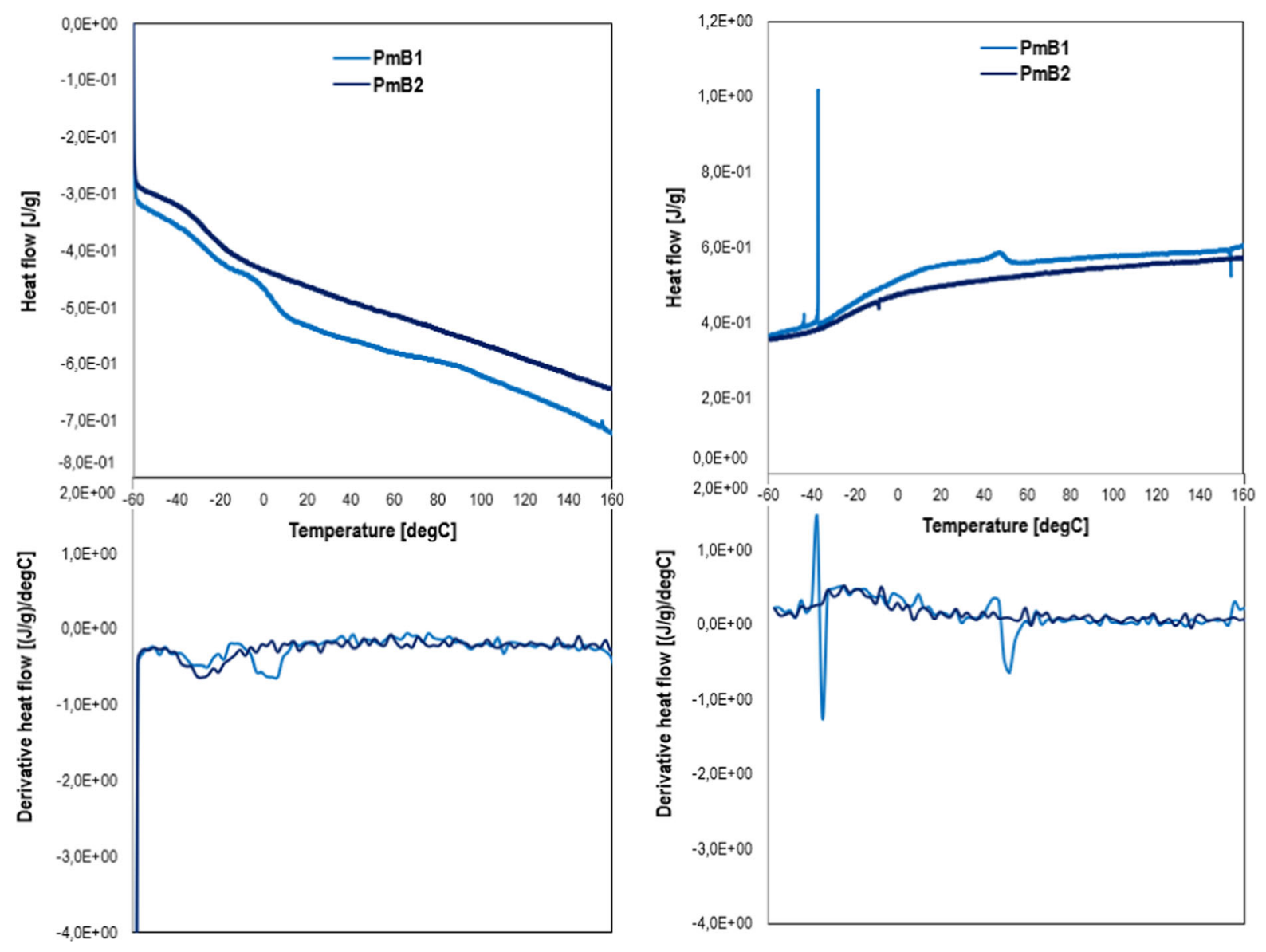

Fig. 4 Graphs of heat flow together with their derivative curves of PmB1 and PmB2 during linearly heating (left) and cooling (right) calorimetric scans (TL-DSC, Lab1)

laboratory determined value and those predicted based on ideal mixing theory (expressed by Eq. 2) is obtained, indicating that the two individual components of PmB2 are not exactly following the blending rule.

\section{Summary and future work}

The RILEM TC 272 PIM-TG1 has initiated an interlaboratory program to evaluate the phase and interphase characteristics of bituminous binders. In the present work, emphasis was laid on comparing the $T_{g}$ measured by conventional temperature linear-DSC (TL-DSC), and temperature modulation-DSC (TMDSC), improving the interpretation of glass transition in binders. To restrain the scope of this study, two SBS polymer modified binders (PmBs) were evaluated and compared with two neat bituminous binders.

The major topics discussed in the research are summarized as follows:

- In TL-DSC technique, the $T_{g}$ values of binders were determined by calculating the maximum of the 1st derivative of the heating heat flow curve. According to the two implemented protocols, there was a slight reduction in heat flow baseline after the glass transition. The appearance of the second peak of the 1st derivative of heat flow in PmB1 may result from a cold crystallisation. Despite the cold crystallisation after the glass transition in PmB1, the PmB2 did not show a second peak of the 1 st heat flow derivative or any deviation on the heat flow baseline after the glass transition.

- In TM-DSC technique, two temperature modulation protocols with the same heating rate were 

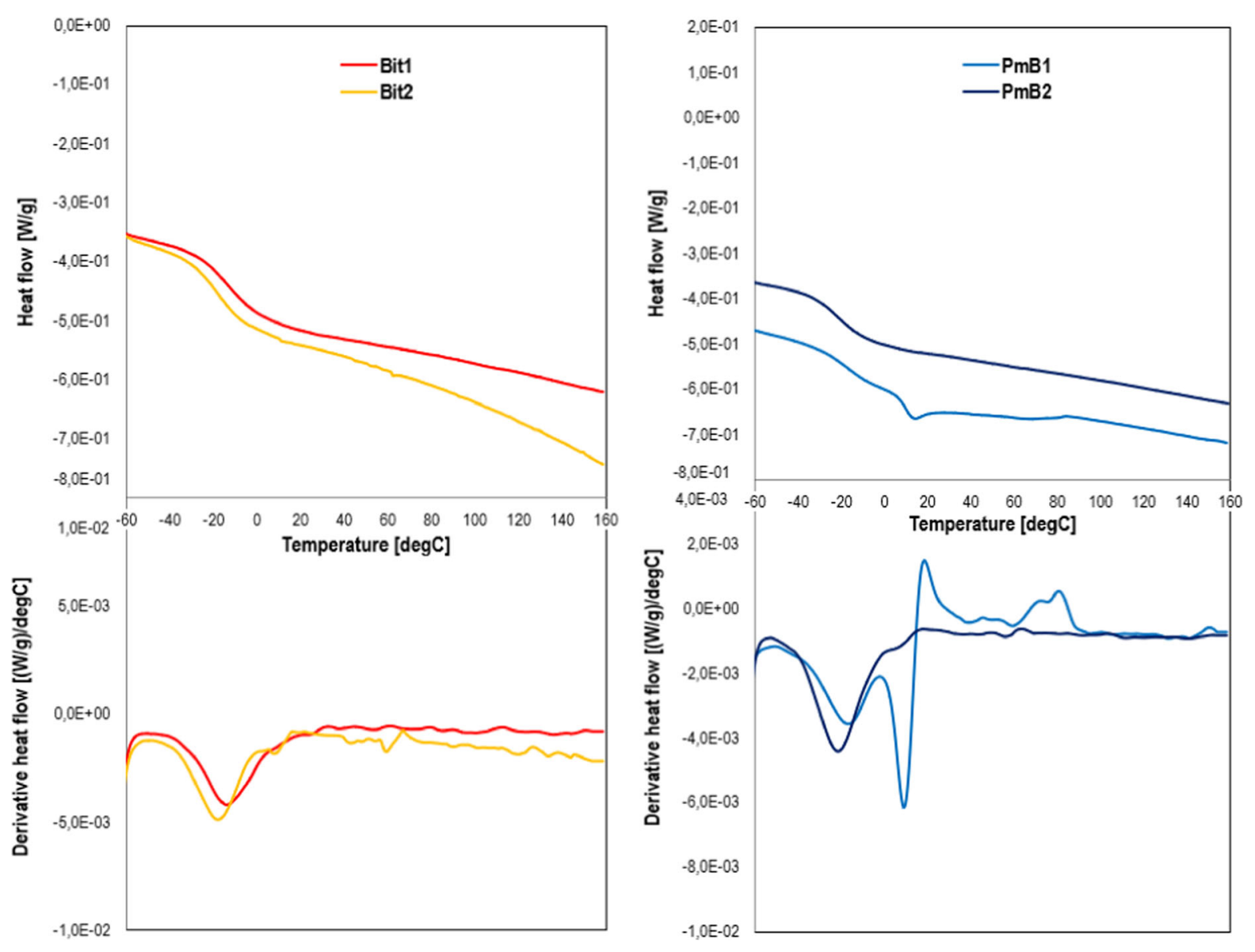

Fig. 5 Graphs of heat flow together with their derivative curves of Bit1 and Bit2 (left), and PmB1 and PmB2 (right) during linearly heating calorimetric scans (TL-DSC, Lab12)

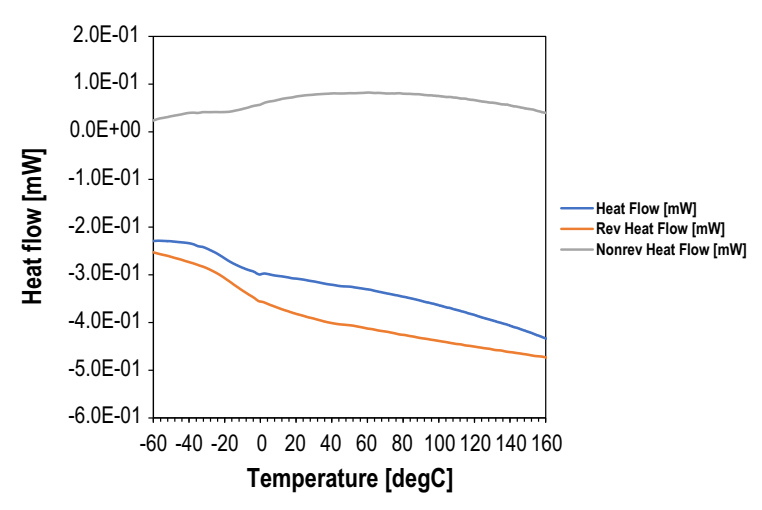

Fig. 6 Total, reversing and non-reversing heat flow of Bit1 during heating (TM-DSC, Lab2)

implemented. It was noticed that the reversing heat capacity signals of PmB1 did not show a second peak of the 1st derivative as observed in TL-DSC heating scans. The TM-DSC technique minimized cold crystallisation by separating structural relaxation phenomena from the total heat capacity measurements. The structural relaxation did not significantly influence the glass transition detection based on the reversing component of heat capacity from the TM-DSC heating scans due to the underlying heating. This study confirmed that the TM-DSC is a more robust and precise technique than the TL-DSC.

- The bitumen modification by $7.5 \mathrm{wt} \%$ vinyl SBS led to the $T_{g}$ reduction reflecting the positive influence of SBS at low temperatures.

Further research is needed to obtain an in-depth understanding of the effect of chemical composition on the thermal behavior of bituminous binders using DSC. Some recommendations for future 
Fig. 7 Graphs of Bit1 and Bit2; a heat flow together with their derivative curves during linear temperature cooling (TL-DSC), and b total heat capacity and c reversible heat capacity with their derivative curves during temperature modulation heating (TMDSC, Lab2)

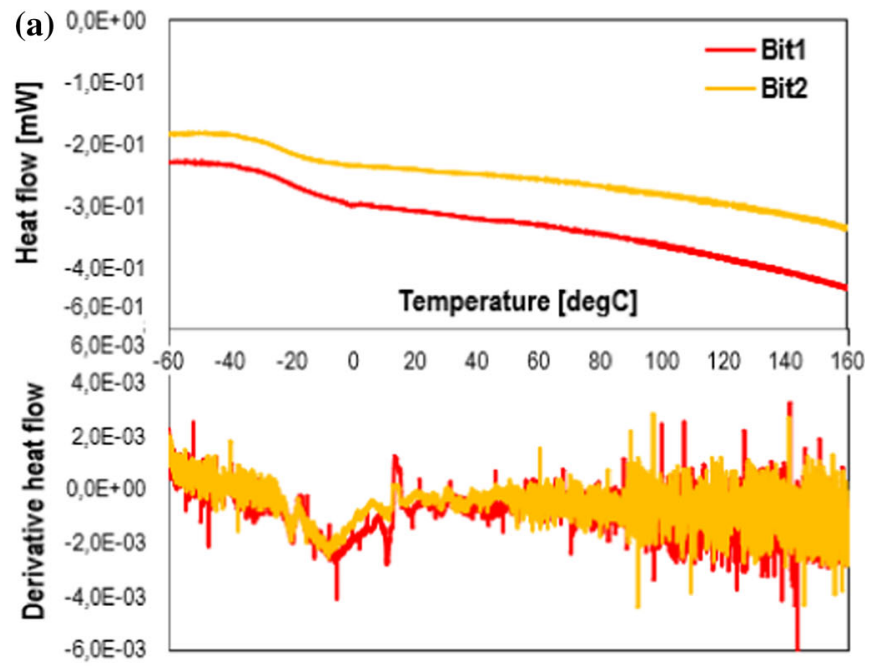

(b)

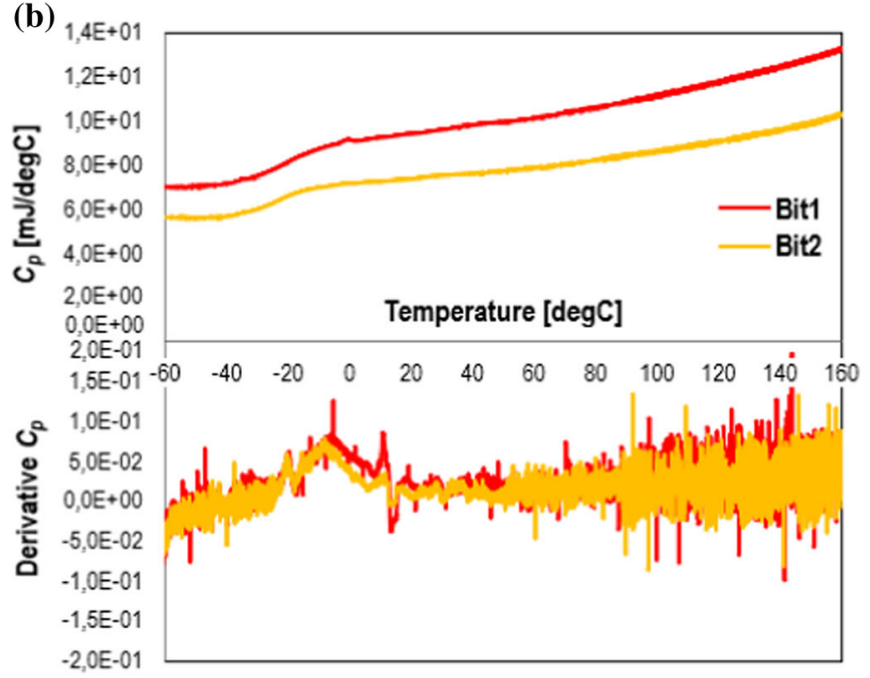

(c)

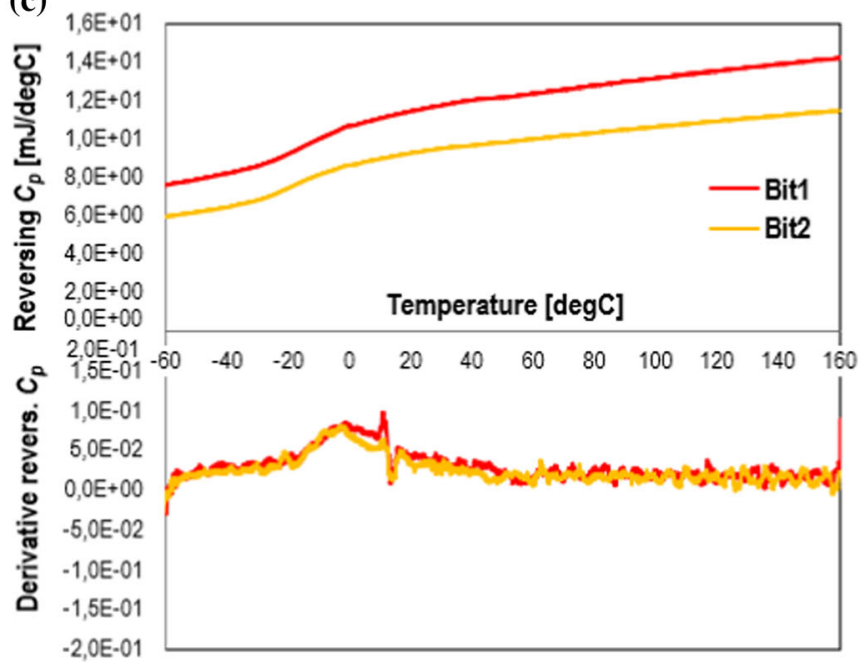


Fig. 8 Graphs of PmB1 and PmB2; a heat flow together with their derivative curves during linear temperature cooling (TL-DSC), and b total heat capacity and c reversible heat capacity with their derivative curves during temperature modulation heating (TMDSC, Lab2)

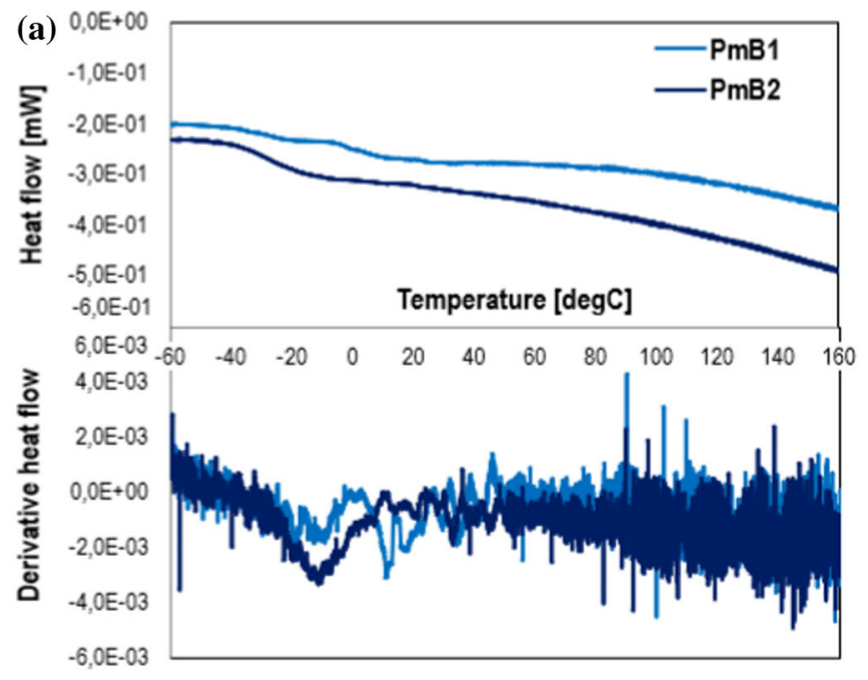

(b) $1,6 \mathrm{E}+01$
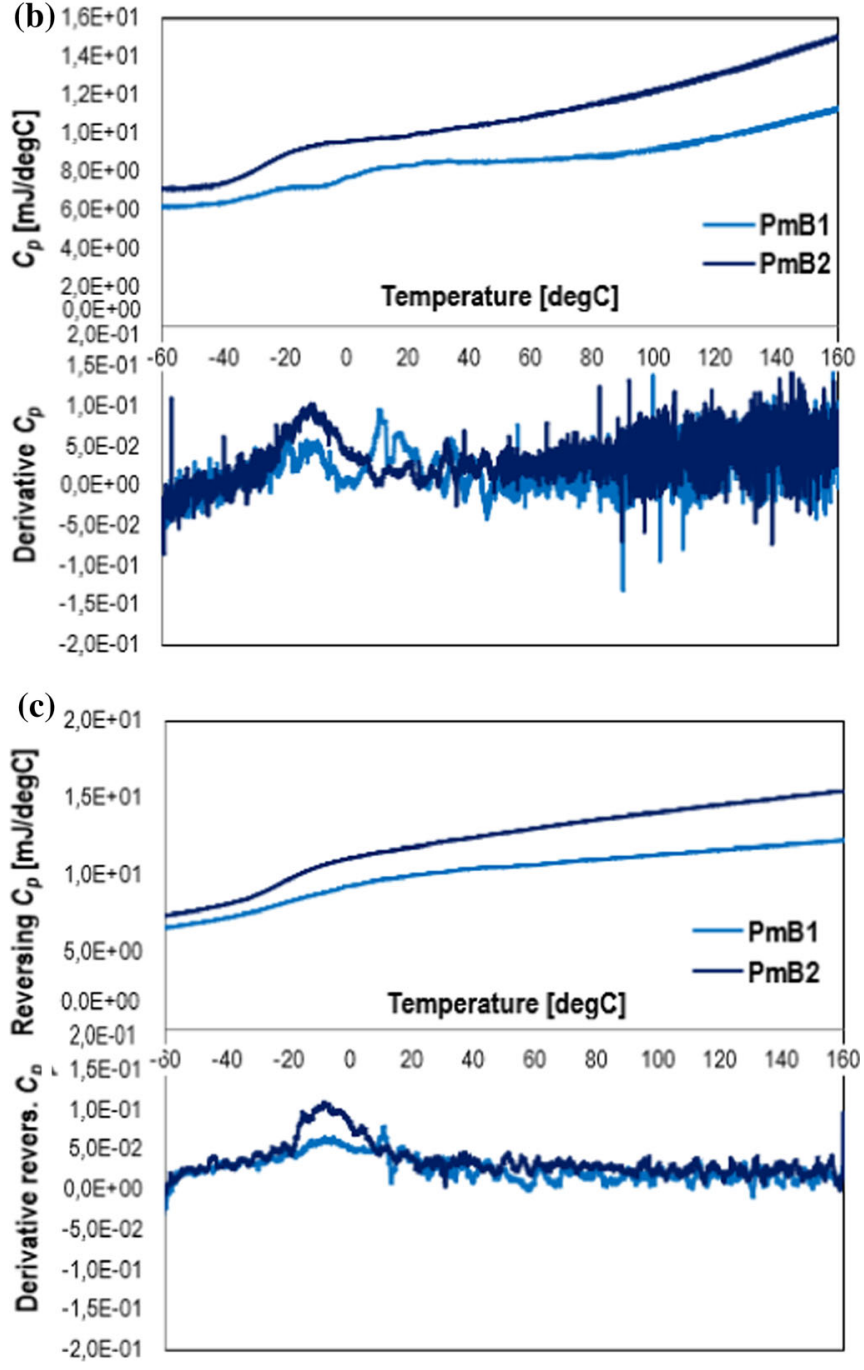

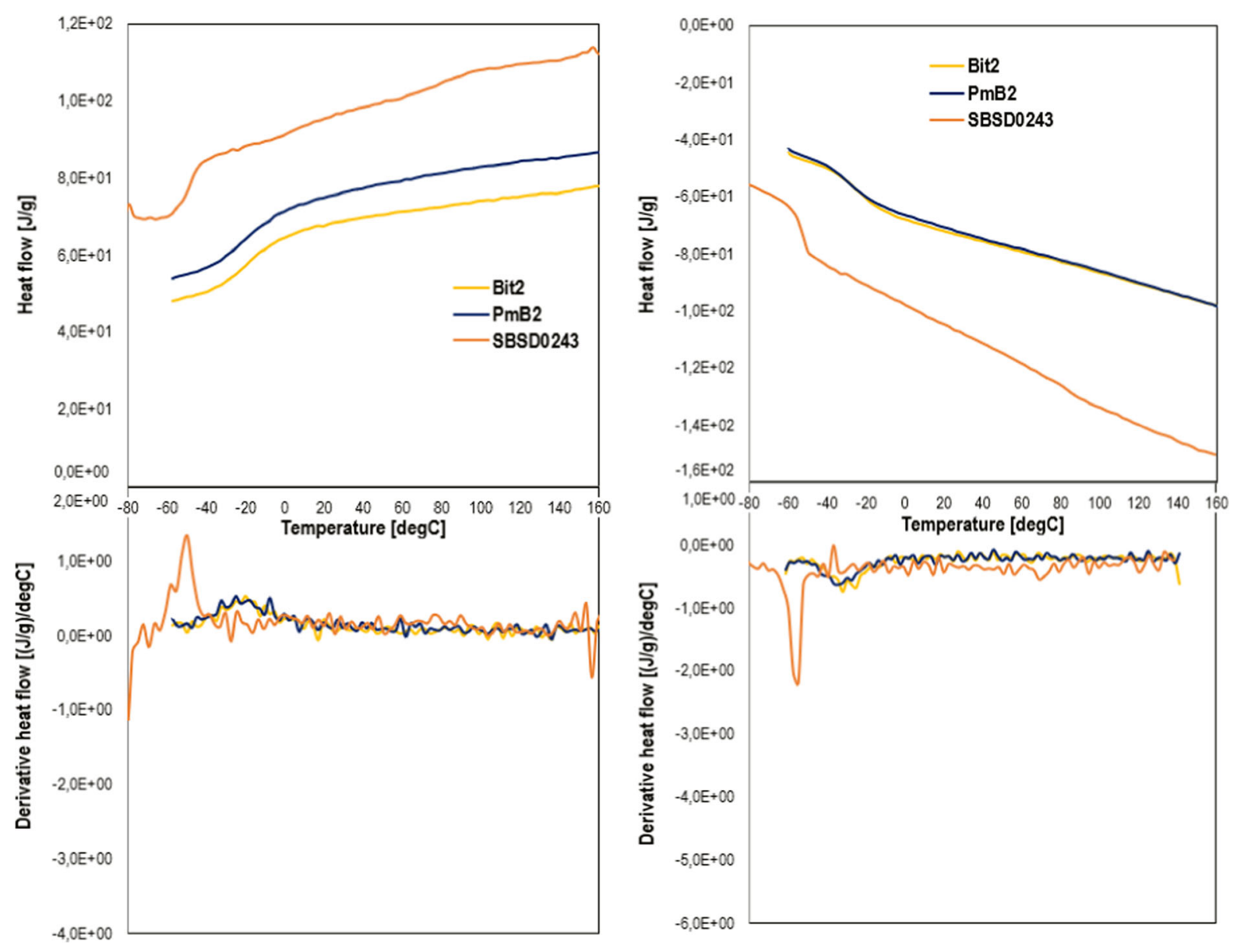

Fig. 9 Graphs of heat flow together with their derivative curves of Bit2, SBS polymer, and PmB2 (i.e., Bit2 together with 7.5\% of SBS polymer) during linearly heating (left) and cooling (right) calorimetric scans (TL-DSC, Lab1)

studies to characterise such multiphase systems are as follows:

- Binders from different origins may show different DSC thermal responses mainly due to variations in their chemical composition. As the fractions of saturates, aromatics, resins, and asphaltenes and their functional groups determine the chemistry of bitumen, it would be beneficial to conduct both TLDSC and TM-DSC analyses in the individual bituminous fractions to reveal their thermo-kinetics characteristics.

- The current research presented the influence of SBS on the glass transition in bituminous binders. Since various additives and modifiers are available to enhance the properties of binders, the investigation of their effect on the thermal behavior of bituminous binders is critical as well.
- The Fox equation was used in this study to predict the ideal mixing glass transition of a PmB. However, different additives and modifiers demonstrate significantly different affinities with binders. Therefore, the applicability of other prediction schemes of the composition dependence of the $T_{g}$ of modified binders should be assessed in a future research.

- The correlation of glass transition temperature with rheological properties could be established to obtain a comprehensive picture of the thermorheology of bituminous binders.

\section{Declarations}

Conflict of interest The authors declare that they have no conflict of interest. 
Open Access This article is licensed under a Creative Commons Attribution 4.0 International License, which permits use, sharing, adaptation, distribution and reproduction in any medium or format, as long as you give appropriate credit to the original author(s) and the source, provide a link to the Creative Commons licence, and indicate if changes were made. The images or other third party material in this article are included in the article's Creative Commons licence, unless indicated otherwise in a credit line to the material. If material is not included in the article's Creative Commons licence and your intended use is not permitted by statutory regulation or exceeds the permitted use, you will need to obtain permission directly from the copyright holder. To view a copy of this licence, visit http://creativecommons.org/licenses/by/4.0/.

\section{References}

1. Claudy P, Letoffe JM, King GN, Planche JP (1992) Characterization of asphalts cements by thermomicroscopy and differential scanning calorimetry: correlation to classic physical properties. Fuel Sci Technol Int 10(4-6):735-765

2. Harrison IR, Wang G, Hsu TC (1992) A differential scanning calorimetry study of asphalt binders. Strategic Highway Research Program (SHRP). National Academy of Sciences: Washington, D.C. SHRP-A/UFR-92-612

3. Turner TF, Branthaver JF (1997) DSC studies of asphalt and asphalt components. Asphalt Science and Technology, Marcel Dekker Inc., pp 59-101

4. Robertson RE, Branthaver JF, Harnsberger PM, Petersen PC, Dorrence SM, McKay JF, Turner TF, Pauli AT, Huang SC, Huh JD, Tauer JE (2001) Fundamental properties of asphalts and modified asphalts, volume I: interpretive report. Report No. FHWA-RD-99-212. Western Research Institute

5. Masson JF, Polomark GM (2001) Bitumen microstructure by modulated differential scanning calorimetry. Thermochim Acta 374:105-114

6. Gill PS, Sauerbrunn SR, Reading M (1993) Modulate differential scanning calorimetry. J Therm Anal 40:931-939

7. Schawe JEK (1995) A comparison of different evaluation methods in modulated temperature DSC. Thermochim Acta 260:1-16

8. Reading M (2001) The use of modulated temperature programs in thermal methods. J Therm Anal Calorim 64:7-14

9. Simon SL (2001) Temperature-modulated differential scanning calorimetry: theory and application. Thermochim Acta 374:55-71

10. Frolov IN, Yusupova TN, Ziganshin MA, Okhotnikova ES, Firsin AA (2017) Dynamics of formation of asphalt microstructure according to modulated differential scanning calorimetry data. Pet Chem 57(12):1002-1006

11. Qiu Y, Ding H, Zheng P (2020) Toward a better understanding of the low-temperature reversible aging phenomenon in asphalt binder. Int J Pavem Eng. https://doi.org/ 10.1080/10298436.2020.1740926

12. Masson JF, Bundalo-Perc S, Delgado A (2005) Glass Transitions and mixed phases in block SBS. J Polym Sci Part B Polym Phys 43:276-279
13. Khan AN, Hesp SAM (2019) Comparison between thermal, rheological and failure properties for the performance grading of asphalt cements. Constr Build Mater 220:196-205

14. Chailleux E, Queffélec C, Borghol I, Farcas F, Marceau S, Bujoli B (2021) Bitumen fractionation: contribution of the individual fractions to the mechanical behavior of road binders. Constr Build Mater 271:121528

15. Cheung CY, Cebon D (1997) Deformation mechanisms of pure bitumen. J Mater Civ Eng 9(3):117-129

16. Masson JF, Polomark GM, Collins P (2002) Time-dependent microstructure of bitumen and its fractions by modulated differential scanning calorimetry. Energy Fuels $16: 470-476$

17. Lesueur D (2009) The colloidal structure of bitumen: consequences on rheology and on the mechanism of bitumen modification. Advances in Colloidal and Interface Science 145:42-82

18. Redelius P, Soenen H (2015) Relation between bitumen chemistry and performance. Fuel 140:34-43

19. Letoffe JM, Claudy P, Kok MV, Garcin M, Volle JL (1995) Crude oils: characterization of waxes precipitated on cooling by DSC and thermomicroscopy. Fuel 74:810-817

20. Claudy PM, Letoffe PM, Martin D, Planche JP (1998) Thermal behavior of asphalt cements. Thermochim Acta 324:203-213

21. Chambrion P, Bertau R, Ehrburger P (1996) Characterization of bitumen by differential scanning calorimetry. Fuels 75(2):144-148

22. Lu X, Redelius P (2007) Effect of bitumen wax on asphalt mixture performance. Constr Build Mater 21:1961-1970

23. Kriz P, Stastna J, Zanzotto L (2007) Glass transition and phase stability in asphalt binders. Road Mater Pavem Design 9:37-65

24. Planche JP, Claudy PM, Letoffe JM, Martin D (1998) Using thermal analysis methods to better understand asphalt rheology. Thermochim Acta 324(1):223-227

25. Elwardany MD, Planche JP, Adams JJ (2019) Determination of binder glass transition and crossover temperatures using 4-mm plates on a dynamic shear rheometer. Transp Res Rec 2673(10):247-260

26. Bahia H (1992) Low-temperature isothermal physical hardening of asphalt cements. PhD dissertation. The Pennsylvania State University

27. Anderson DA, Marasteanu MO (1999) Physical hardening of asphalt binders relative to their glass transition temperatures. Transp Res Rec 1661:27-34

28. Elwardany M, King G, Planche J-P, Rodezno C, Christensen D, Fertig RS III, Kuhn KT, Bhuiyan FH (2019) Internal restraint damage mechanism for age-induced pavement surface distresses: block cracking and raveling. J Assoc Asphalt Paving Technol 88:1-47

29. Elwardany M, Planche JP, King G (2020) Universal and practical approach to evaluate asphalt binder resistance to thermally-induced surface damage. Constr Build Mater 255:119331

30. Ho RM, Adedeji A, Giles DW, Hajduk DA, Macosko CW, Bates FS (1997) Microstructure of triblock copolymers in asphalt oligomers. J Polym Sci Part B Polym Phys $35: 2857-2877$ 
31. Fawcett AH, McNally T (2001) Blends of bitumen with polymers having a styrene component. Polym Eng Sci 41(7):1251-1264

32. Kamiya S, Tasaka S, Zhang X, Dong D, Inagaki N (2001) Compatibilizer role of styrene-butadiene-styrene triblock copolymer in asphalt. Polym J 33(3):209-213

33. Varma R, Takeichi H, Hall JE, Ozawa YF, Kyu T (2002) Miscibility studies on blends of kraton block copolymer and asphalt. Polymer 43:4667-4671

34. Masson JF, Polomark G, Collins P (2005) Glass transitions and amorphous phases in sbs-bitumen blends. Thermochim Acta 436:96-100

35. Adams JJ, Elwardany MD, Planche J-P, Boysen RB, Rovani JF (2019) Diagnostic techniques for various asphalt refining and modification methods. Energy Fuels 33:2680-2698
36. Porot L, Chailleux E, Apostolidis P, Zhu J, Margaritis A, Tsantilis L (2020) Complex bituminous binders, are current test methods suitable for?. RILEM International Symposium on Bituminous Materials, Lyon, France

37. Fox TG (1956) Influence of diluent and copolymer composition on the glass temperature of a polymer system. Bull Am Phys Soc 1:123

Publisher's Note Springer Nature remains neutral with regard to jurisdictional claims in published maps and institutional affiliations. 